IN BRIEF

IB D

Phase II trial success for anti-MADCAM1 antibody

Many patients with ulcerative colitis are refractory towards conventional therapies, highlighting a need for treatments with novel mechanisms of action. Promising targets include cell adhesion molecules such as MADCAM1 that mediate the migration of lymphocytes into sites of inflammation in the gut. In a phase II, randomized, placebo-controlled trial, the efficacy and safety of PF-00547659 - a human monoclonal antibody to MADCAM1 - was assessed in moderate to severe ulcerative colitis. Patients $(n=357)$ from 105 centres in 21 countries were randomized to receive placebo or one of four doses of PF-00547659 every 4 weeks. The primary endpoint was induction of clinical and endoscopic remission by week 12 . In three of the four treatment groups $(7.5 \mathrm{mg}, 22.5 \mathrm{mg}$ and $75 \mathrm{mg}$ ), remission rates were significantly greater than placebo, with the greatest clinical effects seen with doses of $22.5 \mathrm{mg}$ and $75 \mathrm{mg}$. No safety or tolerance concerns were reported

ORIGINAL ARTICLE Vermeire, S. et al. Anti-MAdCAM antibody (PF-00547659) for ulcerative colitis (TURANDOT): a phase 2, randomised, double-blind, placebo-controlled trial. Lancet http://dx.doi.org/10.1016/S0140-6736(17)30930-3 (2017)

\title{
THERAPY
}

\section{Bacteriophages important for FMT efficacy}

Faecal microbiota transplantation (FMT) is effective for patients with recurrent Clostridium difficile infection (CDI). However, the mechanisms of FMT remain poorly understood and, despite data suggesting that bacteriophages influence the success of FMT, data on viral alterations in $\mathrm{CDI}$ and their effects on clinical outcome are scarce. In a preliminary study, Zuo et al. used ultra-deep metagenomic sequencing to compare enteric virome changes in patients with $\mathrm{CDI}(n=24)$ with healthy controls $(n=20)$, longitudinally assessing alterations in nine patients after FMT. Patients with CDI were found to have a higher abundance but lower diversity of the bacteriophage Caudovirales compared with controls, suggesting enteric virome dysbiosis in CDI. Furthermore, cure after FMT was more likely when an increased fraction of the recipient enteric virome was occupied by donor-derived Caudovirales taxa, suggesting that bacteriophages might play a role in FMT efficacy.

ORIGINAL ARTICLE Zuo, T. et al. Bacteriophage transfer during faecal microbiota transplantation in Clostridium difficile infection is associated with treatment outcome. Gut http://dx.doi.org/10.1136/gutinl-2017-313952 (2017)

\section{$\Rightarrow$ PRIMARY SCLEROSING CHOLANGITIS} Ursodeoxycholic acid derivative: safe and effective

Primary sclerosing cholangitis (PSC) is a chronic cholestatic disease with no effective medical therapy. A homologue of ursodeoxycholic acid, 24-norursodeoxycholic acid (norUDCA), was previously shown to be effective in mouse models of cholestatic and fibrotic liver diseases, prompting a randomized, placebo-controlled trial to evaluate its efficacy and safety for PSC. In the study, conducted in 38 centres from 12 countries, 161 patients with $\mathrm{PSC}$ were randomized to receive placebo or one of three doses ( $500 \mathrm{mg}, 1,000 \mathrm{mg}$ or $1,500 \mathrm{mg}$ per day) of norUDCA for 12 weeks. The primary efficacy endpoint was the relative change in serum alkaline phosphatase (ALP) levels between baseline and final visit. A significant dose-dependent decrease in ALP levels was seen in response to all treatments with norUDCA. Importantly, the safety profile of norUDCA was similar to placebo, justifying further trials in PSC.

ORIGINAL ARTICLE Fickert, P. et al. norUrsodeoxycholic acid improves cholestasis in primary sclerosing cholangitis. J. Hepatol. http://dx.doi.org/10.1016/j.jhep.2017.05.009 (2017) 